\title{
Comparison of the normal sinus node with seven types of rate responsive pacemaker during everyday activity
}

\author{
A N Sulke, A Pipilis, R A Henderson, C A Bucknall, E Sowton
}

\section{Abstract}

The heart rate response of 59 patients aged 17-79 years implanted with seven different types of rate responsive pacemakers was evaluated during graded exercise treadmill testing and during standardised daily activities. The heart rate response in patients with pacemakers was compared with the chronotropic response in 20 healthy controls of similar age and sex distribution who performed identical protocols. All pacemaker types adequately simulated the control heart rate response during the graded exercise treadmill test except during the early stages of exercise. However, during everyday activities, the response of ventricular rate responsive (VVIR) pacemakers was varied. Activity sensing systems rapidly overresponded to staircase descent, to changes in walking speed, and to suitcase lifting with the pacemaker arm, and these systems did not respond to mental stress. "Physiological" sensors (QT and minute ventilation units) responded slowly to rapid changes in physiological demand. The QT pacemaker patients did respond to mental stress but showed a paradoxical increase in rate during the recovery phases of burst exercise protocols such as staircase ascent/descent and walking deceleration. Dual chamber pacemakers in VDD, DDD, and DDDR modes most closely simulated the normal chronotropic response during everyday activities.

Graded exercise treadmill testing, in isolation, may not be the best way to assess or program the heart rate response in patients with rate adaptive pacemakers because changes in heart rate during everyday activities may deviate considerably from the normal sinus response despite satisfactory simulation of the normal chronotropic response during treadmill testing.

A N Sulke
A Pipilis

R A Henderson

C A Bucknall

E Sowton

Correspondence to

Dr A N Sulke,

Department of Cardiology,

Guy's Hospital, St Thoma

Accepted for publication

12 February 1990

Pacemaker implantation, as well as increasing life expectancy by preventing Adams-Stokes seizures and life threatening bradycardias,' can also improve the patient's quality of life by increasing physical capability. ${ }^{2}$ Rate res- ponsive pacing systems are now increasingly used to achieve this end.

The advantages of single chamber rate responsive pacing over VVI pacing are now well established..$^{3-7}$ Several different types of rate responsive pacemakers are currently available (both single and dual chamber) with various sensors including those that detect activity, ${ }^{8}$ minute ventilation, ${ }^{9}$ QT interval, ${ }^{10}$ central venous temperature, ${ }^{11}$ venous oxygen saturation, ${ }^{12} \mathrm{pH}$ value, ${ }^{13}$ and, more recently, $\mathrm{dP} / \mathrm{dt}^{14}$ and the integral of the evoked response. ${ }^{15}$

Most previous studies to assess sensor driven rate responsive pacemakers used treadmill testing or bicycle ergometry. ${ }^{16-18} \mathrm{We}$ compared heart rate changes in patients with single and dual chamber rate responsive pacemakers with the chronotropic response in a group of healthy controls during various standardised everyday activities and during graded exercise treadmill tests.

\section{Patients and methods}

PATIENTS

Fifty nine patients aged 17-79 years (mean $57.9,35\left(59^{\circ}{ }_{0}\right)$ men) had seven types of rate responsive pacemaker implanted. Ventricular rate responsive pacemakers (VVIR) were implanted in 33 patients. They were the activity sensing Siemens Sensolog I (10), minute ventilation sensing Telectronics Meta (8), activity sensing Medtronic Activitrax (8), and QT sensing Vitatron Tx II (7). None of these patients had the signs or symptoms of the pacemaker syndrome. ${ }^{19}$

Dual chamber rate responsive systems were implanted in 26 patients. Six had Medico Phymos VDD systems, which require a single lead with free floating atrial bipolar sensing and unipolar ventricular sensing and pacing; 10 had DDD systems; and 10 had activity sensing DDDR systems. The table shows the indications for implantation.

All patients were followed up at 6 weeks and 3 months after pacemaker implantation and were optimally programmed to the rate response mode according to the manufacturers' instructions.

We studied heart rate responses in a group standardised daily activities and treadmill testing. years (mean $55.0,12(60 \%)$ men) during 
Patient details and indication for pacemaker implantation

\begin{tabular}{|c|c|c|c|c|c|}
\hline Pacemaker type & Mode & No & No male $(\%)$ & Age range (mean) & Indication for implant \\
\hline $\begin{array}{l}\text { Telectronic Meta } \\
\text { Medtronic Activitrax } \\
\text { Siemens Sensolog } \\
\text { Vitatron Tx II } \\
\text { Medico Phymos } \\
\text { Various } \\
\text { Siemens Multilog (3) Pacesetter Synchrony (7) }\end{array}$ & $\begin{array}{l}\text { VVIR } \\
\text { VVIR } \\
\text { VVIR } \\
\text { VVIR } \\
\text { VDD } \\
\text { DDD } \\
\text { DDDR }\end{array}$ & $\begin{array}{r}8 \\
8 \\
10 \\
7 \\
6 \\
10 \\
10\end{array}$ & $\begin{array}{l}2(25) \\
1(12 \cdot 5) \\
8(80) \\
5(71) \\
5(83) \\
8(80) \\
6(60)\end{array}$ & $\begin{array}{l}38-79(63 \cdot 3) \\
22-77(58 \cdot 9) \\
43-73(55 \cdot 8) \\
52-69(57 \cdot 9) \\
52-78(62 \cdot 1) \\
22-77(59 \cdot 6) \\
17-68(50 \cdot 6)\end{array}$ & $\begin{array}{l}\text { PHA(2),B(2),AVII + AVIII(4) } \\
\text { PHA(3),AF + B(1),SSS(2),AVII + AVIII(2) } \\
\text { PHA(5),AF + B(1),SSS(1),B(1),AVII + AVIII(2) } \\
\text { PHA(2),AF + B(2),SSS(2),AVII + AVIII(1) } \\
\text { AVII(2),AVIII(4) } \\
\text { B(1),SSS + AVII(1),AVII(2),AVII + AVIII(6) } \\
\text { SSS + AVII + AVIII(8),AVII + AVIII(2) }\end{array}$ \\
\hline Total & & 59 & $35(59)$ & $17-79(57 \cdot 9)$ & \\
\hline
\end{tabular}

PHA, post His bundle ablation; B, symptomatic bradycardia; AF, slow atrial fibrillation; SSS, sick sinus syndrome; AVII, 2nd degree atrioventricular block (Mobitz type II); AVIII, complete atrioventricular block.

HEART RATE MONITORING

During the study continuous six lead electrocardiograms were recorded with a Siemens Mingolog 7 at a paper speed of $25 \mathrm{~mm} / \mathrm{s}$. The heart rate was calculated from the mean $R R$ interval of the five consecutive beats recorded immediately after each stage was completed. Before each activity protocol the resting heart rate was measured after the individual had sat at rest for 2 minutes.

STUDY PROTOCOLS: GRADED TREADMILL TEST Patients performed graded exercise treadmill testing according to the Chronotropic Assessment Exercise Protocol (CAEP) ${ }^{20}$ All individuals were exercised to peak effort tolerance.

\section{POSTURE CHANGE}

The effects of changes in posture on heart rate were determined by measuring mean RR interval over five beats when the individual was lying, seated, and standing. Heart rate was measured after individuals had rested supine on a standard examination couch for 2 minutes. The heart rate was measured immediately after the subjects sat upright and immediately they stood up.

\section{MENTAL STRESS}

The effect of mental stress on heart rate was determined over a 2 minute period during which the individual was asked to subtract 7 from 100 serially aloud. The supervising clinician vigorously encouraged the subject throughout the study period. Heart rate was measured at rest before mental stress, after 60 seconds of stress, and after 120 seconds of stress (that is, immediately after it stopped.

\section{SUITCASE LIFTING}

The subjects were asked to lift a standard suitcase $(45 \mathrm{~cm} \times 35 \mathrm{~cm} \times 12 \mathrm{~cm}$ weighing $10 \mathrm{~kg}$ ) with both hands from the floor on to the examination couch (a height of $82 \mathrm{~cm}$ ) and then immediately back on to the floor. Heart rate was measured before and immediately after they lifted the suitcase.

Subjects were then randomly assigned to suitcase lifting with either the right or left arm and instructed to raise the case from the floor to above hip height four times without stopping and heart rate was assessed at rest and after the procedure. The subject was rested until the basal heart rate was achieved and they repeated the suitcase lifts with the opposite arm.

\section{CHANGES IN WALKING SPEED}

We assessed the response of heart rate to rapid acceleration and deceleration and talking while walking.

Subjects were rested until basal heart rate was attained (stage 0). They were then instructed to walk with normal arm movement on a treadmill at $1 \mathrm{mph}(1.6 \mathrm{~km} / \mathrm{h})$ and $0^{\circ}$ gradient for 30 seconds (stage 1). They were then engaged in active conversation (discussing their travel plans to and from the study centre) while walking normally at this treadmill rate for 30 seconds (stage 2). Treadmill speed was gradually accelerated over the next 30 seconds to reach $4 \mathrm{mph}(6.4 \mathrm{~km} / \mathrm{h}$ ) (stage 3 ). This rate was maintained for a further 30 seconds (stage 4). The treadmill rate was then gradually decelerated to $1 \mathrm{mph}(1.6 \mathrm{~km} / \mathrm{h})$ during the next 30 seconds (stage 5). The treadmill was maintained at $1 \mathrm{mph}(1.6 \mathrm{~km} / \mathrm{h})$ for the final 30 seconds of the procedure (stage 6). Throughout, individuals were encouraged to walk without using the support bars, if possible.

\section{STAIRCASE ASCENT AND DESCENT}

We measured the response of heart rate to staircase ascent and descent from telemetered electrocardiographic recordings from a Hewlett Packard 78571B recorder.

Subjects were asked to climb two flights of stairs (30 steps, each $15 \mathrm{~cm}$ tall, pitch $27 \mathrm{~cm}$ ) as rapidly as possible and to rest in the erect posture at the top of the second flight. Heart rate was measured at rest and every 15 seconds from the start of the ascent.

Once heart rate had returned to resting values individuals were asked to descend the stairs as fast as possible and to rest at the base of the second flight. Heart rate during staircase descent was calculated every 15 seconds from start of the descent.

\section{STATISTICAL ANALYSIS}

Heart rate and percentage change in heart rate were calculated for each individual at every stage of the protocol in the study, where percentage change in heart rate $=$ observed heart rate - resting heart rate $\div$ resting heart rate $\times 100$

Mean heart rate and mean percentage change in heart rate were calculated for each pacemaker type and for the control population for every stage of each protocol.

We used unpaired Student's $t$ tests to measure statistical differences between percentage change in heart rates in the pacemaker 
Figure 1 A comparison by the graded exercise treadmill test of seven different types of rate responsive pacemaker.

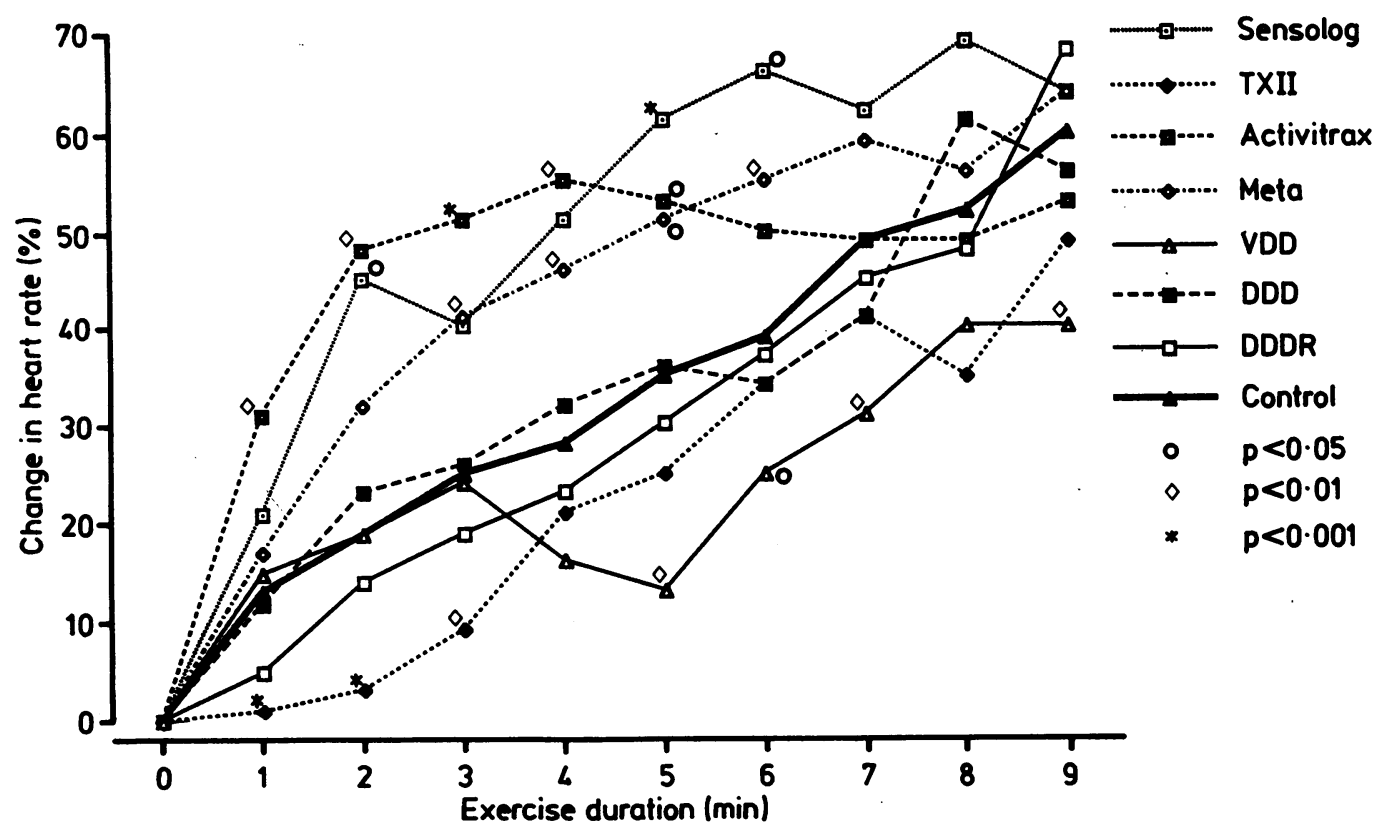

groups and the controls. A p value of $<0.05$ was regarded as statistically significant.

\section{Results}

GRADED EXERCISE TREADMILL TESTS (FIG 1)

During the first 4 minutes of exercise the observed increase in heart rate varied in all single chamber systems. Sensolog, Activitrax, and Meta significantly overresponded while Vitatron $T \mathbf{x}$ patients were significantly ( $p<0.001)$ underresponsive compared with the value in the controls.

During the latter stages of the exercise test and the recovery phase all pacemakers closely simulated heart rate changes in the controls. During recovery, heart rate fell appropriately in all groups except for Vitatron Tx patients in whom high rates decayed more slowly $(p<0.05)$ than in the controls. The VDD patients showed a significant $(p<0.01)$ underresponse after 5 minutes of exercise because the essential rate limiting programming required a pacemaker Wenckebach 2:3

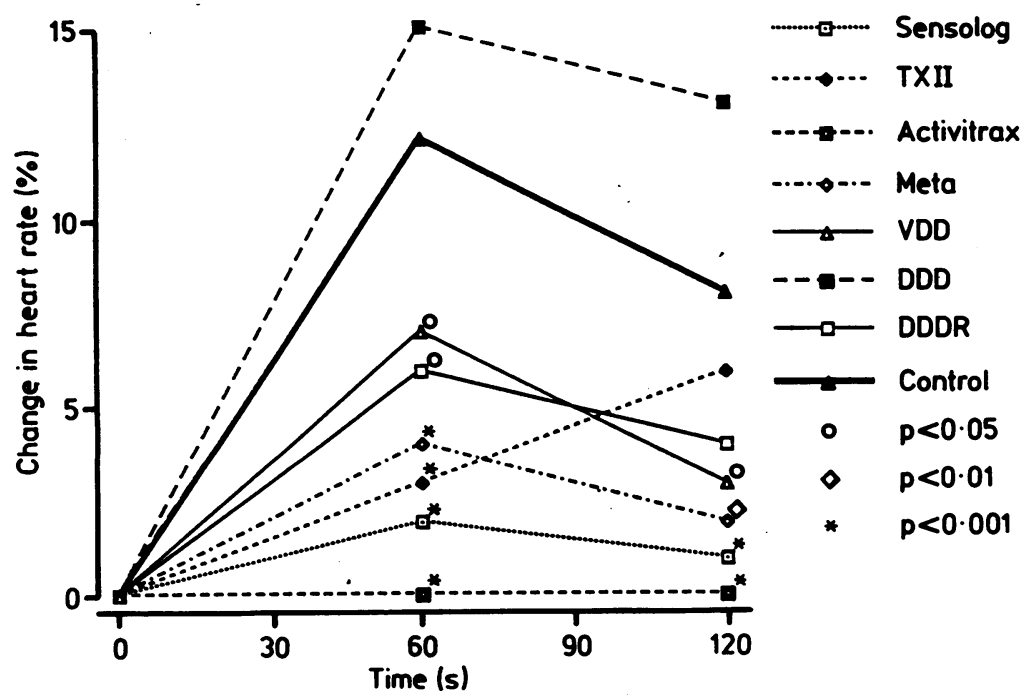

Figure 2 The effect of mental stress on change in heart rate in controls and patients with pacemakers. block to start at comparatively low heart rates unless a very short ( $<100 \mathrm{~ms})$ atrioventricular delay was programmed. This trend occurred in both the other dual chamber modes at high workloads but did not reach statistical significance.

\section{POSTURE CHANGE}

In the controls the heart rate increased when they sat up from the lying position, and increased further when they stood up from the sitting position. The heart rate response with dual chamber and Sensolog pacemakers was similar to that of the controls.

The heart rate response to posture change in patients with Activitrax, Meta, and Vitatron Tx pacemakers was significantly less than that in the controls $(p<0.05, p<0.001$, and $\mathrm{p}<0.001$ respectively).

\section{MENTAL STRESS (FIG 2)}

The increase in heart rate seen during mental stress was similar in patients with DDD pacemakers and controls. Initially the heart rate response in patients with VDD and DDDR pacemakers was significantly less than that in the controls $(p<0.05)$, though at the end of the mental stress test the heart rate response was similar to that of the controls. The Vitatron Tx group showed a delayed heart rate response to mental stress but the mean percentage increase at the end of the stress protocol was similar to that of the controls. Patients with all other pacemakers showed little or no response to mental stress and the changes in heart rate in these patients were significantly less than those in the controls $(\mathrm{p}<0.001)$.

SUITCASE LIFTING (FIG 3)

The increase in heart rate in the controls was similar with four lifts of the right or left arm or a single lift with both arms. All pacemakers performed well except the Vitatron $T x$ which significantly underresponded $(p<0.01)$ to all forms of suitcase lifting. Patients with Sensolog 


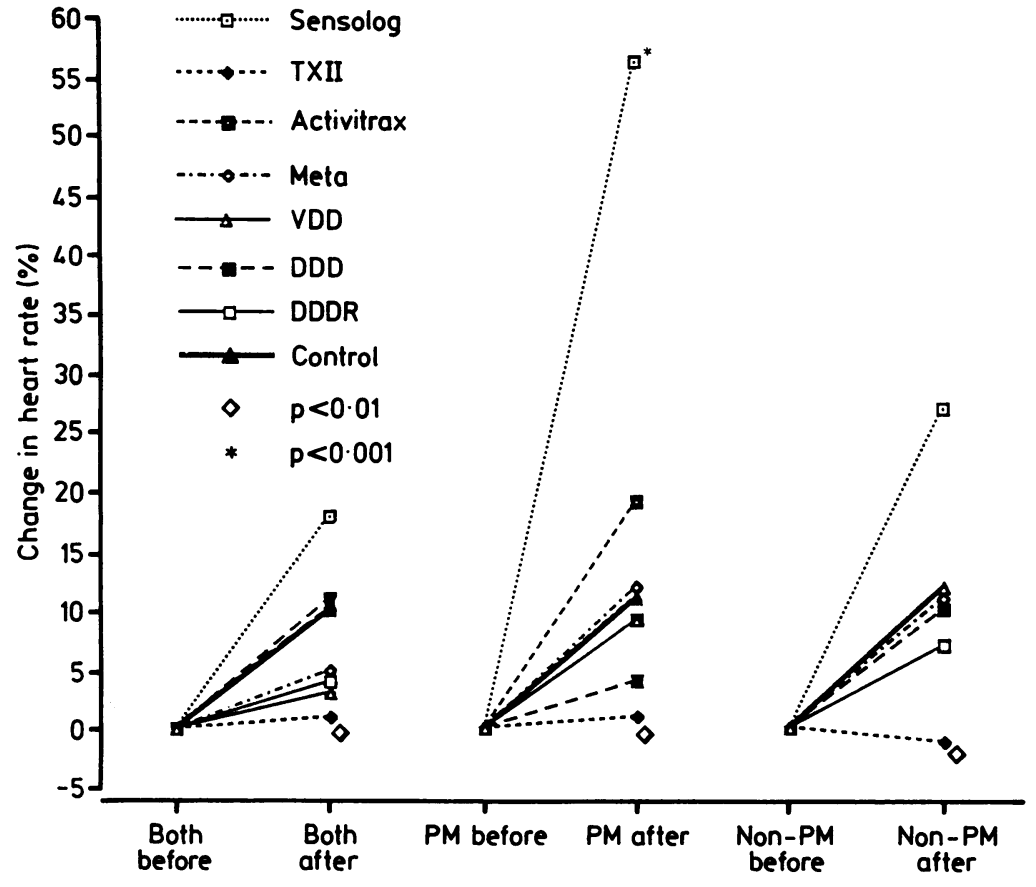

Figure 3 The effect of suitcase lifting on heart rate in controls and patients with pacemakers. Both before, before lifting with both arms; both after, after lifting with both arms; PM before, before lifting with pacemaker arm; PM after, after lifting with pacemaker arm; non-PM before, before lifting with non-pacemaker arm; non-PM after, after lifting with non-pacemaker arm. suitcase lifting with the pacemaker arm $(\mathrm{p}<0.001)$, and patients with Activitrax pacemakers showed a similar trend $(\mathrm{p}<0.08)$.

CHANGE IN WALKING SPEED (FIG 4)

The controls showed a gradual increase in heart rate with a greater increase after stage 4 and a gradual decrease in heart rate after stage 5 . Dual chamber systems closely mimicked the normal sinus response at all stages of this protocol.

Vitatron Tx patients responded slowly to the acceleration phase (stage 3) and fast walking (stage 4) with a paradoxical rate increase during the deceleration phase (stage 5) and final slow walking (stage 6).

The activity sensing VVIR systems produced greater increases in heart rate than were seen in controls $(p<0.001)$ at all stages.

Meta patients overresponded to the acceleration, fast walking, deceleration, and final slow walk (stages 3-6) but the increase in heart rate was appropriate during slow walking and talking.

\section{STAIRCASE ASCENT (FIG 5)}

All individuals completed the ascent within 15 seconds and therefore the remaining 60 seconds of the protocol represents the recovery period. Controls showed a rapid increase in heart rate to a peak after 15 seconds of the protocol, with a plateau at 30 seconds and a gradual decay to resting rate by 75 seconds. Both Meta patients $(p<0.01)$ and Vitatron Tx patients $(\mathrm{p}<0.001)$ underresponded to the initial effort of rapid staircase ascent. Vitatron Tx patients also showed a paradoxical rate increase up to 45 seconds after the initial exertion.

During ascent and recovery, patients with the Activitrax, VDD, DDD, and DDDR systems and controls had similar changes in heart rate.

\section{STAIRCASE DESCENT}

Controls showed half the change in heart rate seen in staircase ascent with a gradual decrease in heart rate from 15 seconds to resting rate by 60 seconds. Activity sensing pacemakers (Sensolog, Activitrax, and DDDR) initially overresponded to rapid staircase descent ( $\mathrm{p}<0.001, \mathrm{p}<0.01$, and $\mathrm{p}<0.05$ respectively). Sensolog patients were slow to return to resting heart rate after the initial exertion.

Vitatron Tx patients underresponded to the
Figure 4 Effect of walking acceleration and deceleration on change in heart rate. Protocol stages are described in text.

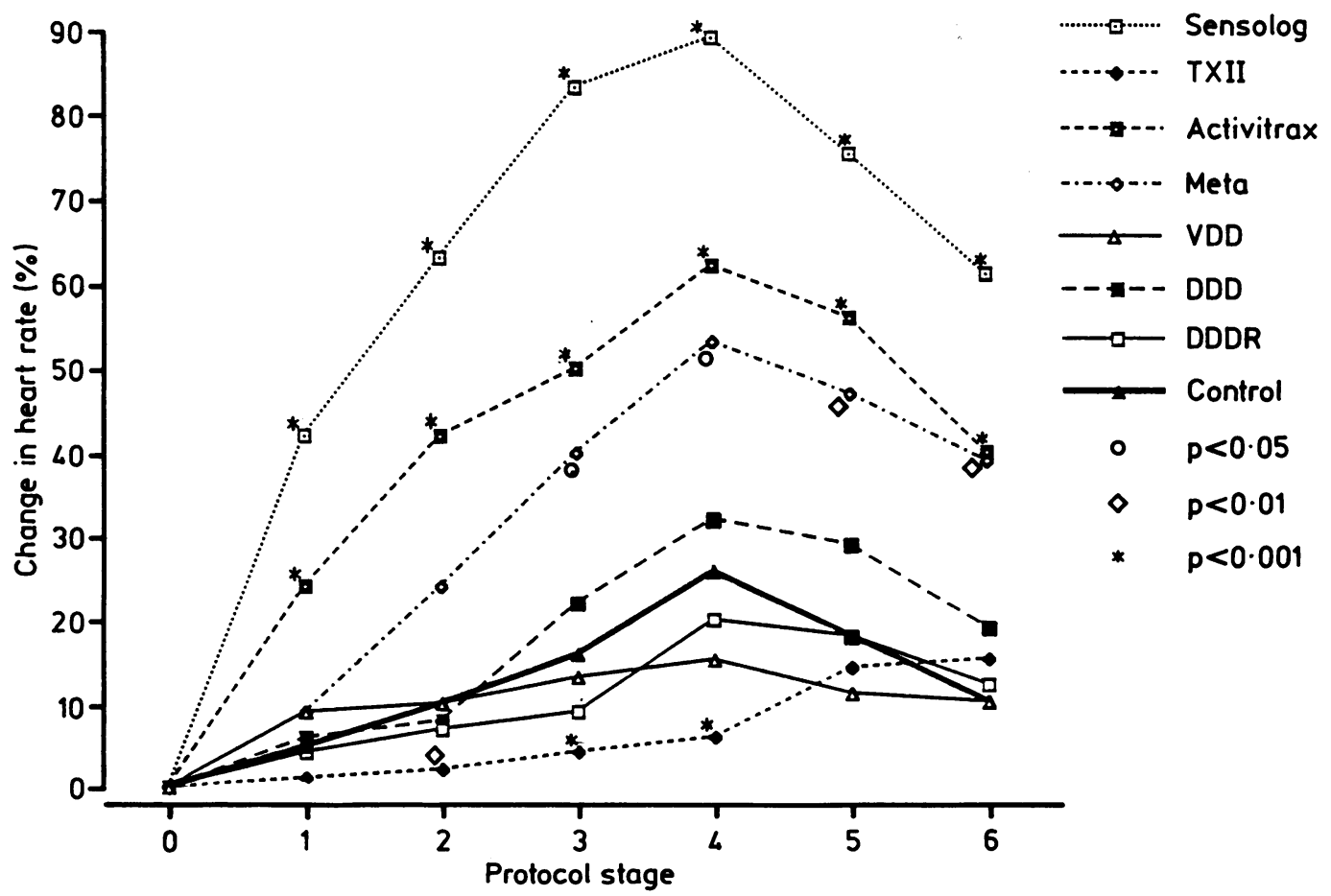


Figure 5 The effect of ascending stairs on change in heart rate.

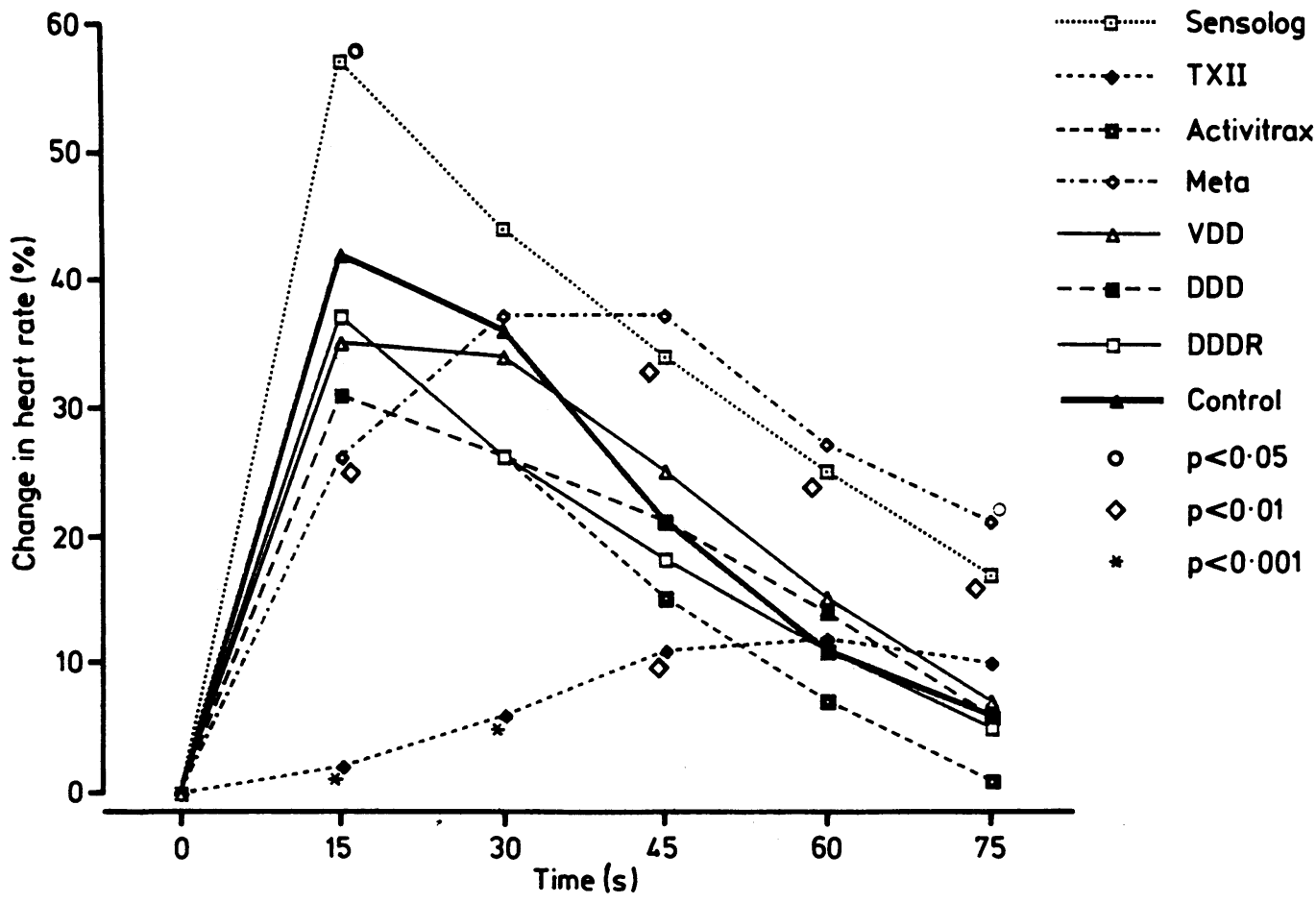

initial effort phase $(p<0.001)$ and showed a paradoxical rate increase in the recovery phase. Patients with Meta and DDD systems showed no significant difference in changes in heart rate compared with the controls.

\section{Discussion}

Previous studies comparing adaptive rate pacing and fixed rate pacing ${ }^{21-23}$ and those comparing different types of rate responsive pacemakers ${ }^{16-18}$ used either treadmill testing or bicycle ergometry to assess haemodynamic function, oxygen consumption', and the heart rate response. In addition, the graded exercise treadmill test is widely used to assess the programming of rate responsive systems. ${ }^{24}$

However, burst exercise is far more common in everyday out of hospital activity than gradually increasing or prolonged physical exertion. Treadmill exercise is also "synthetic" in that the normal movement of the upper body is largely inhibited by both bicycle ergometry and exercise treadmill testing because patients grip either cycle handlebars or treadmill support bars.

We compared the heart rate response of patients with seven different types of rate responsive pacemakers, both dual and single chamber, with the natural chronotropic response of the normal sinus node during various standardised daily activities and during a graded exercise treadmill test. The study was designed to reproduce commonly encountered daily activities that involve burst exercise and also mental stress at rest.

In our patients the paced heart rate response during the graded exercise treadmill tests closely simulated the heart rate response in apparently healthy controls. This is not surprising because rate adaptive pacemakers are developed and evaluated by such tests. These findings confirm those of previous comparative studies, ${ }^{16-18}$ and also suggest that by conventional criteria programming of the pacemakers under study was appropriate. ${ }^{25}$

During the early exercise phase (up to stage 2 , or 4 minutes of exercise) the single chamber units that sensed activity and minute ventilation overresponded while the QT sensing pacemakers underresponded. Dual chamber pacemakers most closely simulated the natural chronotropic response. During the later exercise stages, however, the single chamber units more accurately simulated the normal response. In the dual chamber systems rate limiting programming, which avoids a pacemaker mediated "endless loop" tachycardia by inducing Wenckebach or 2:1 block, caused underresponse at high metabolic demand. These data confirm previous studies of the performance of the single chamber units during treadmill testing. ${ }^{8-10}$

Assessment by the treadmill test indicates that the pacemakers under study are adequate simulators of the normal chronotropic response. The performance of the single chamber units assessed by the daily activity protocols was, however, less satisfactory. During posture change, control heart rates increased more during the change from lying to sitting than from sitting to standing. This was probably because the height of the examination couch meant that subjects required less effort to "stand down" from the seated posture than to rise from the supine to the seated position. The dual chamber systems and the activity sensing Sensolog VVIR pacemakers responded appropriately to this protocol.

The activity sensing units (Sensolog and Activitrax) tended to rapidly overrespond to most of the burst exercise protocols. These were specifically designed to evaluate pacemaker response while subjects were uninhibited by support bars and therefore showed the same movement of the upper body that 
would be encountered during everyday living. The almost universal overresponse suggests that algorithms were designed on the basis of response during "synthetic" exercise such as treadmill exercise or bicycle ergometry. When the patient has uninhibited physical mobility the manufacturers' suggestions for the settings of slope and threshold produce a relative overresponse of paced heart rate for a given physiological demand. There was no significant response to mental, resting stress as would be expected from the sensitivity and specificity of this type of sensor that is described as "non-physiological" by Rossi. ${ }^{26}$ Similarly, lack of heart rate response to the Valsalva manoeuvre and hand grip was shown with activity sensors. ${ }^{27}$ Both Activitrax and Sensolog overresponded to suitcase lifting with the pacemaker arm, but responded appropriately to lifting with both arms and to arm lifting on the non-pacemaker side, again suggesting oversensitivity to movement of the upper torso. The piezo sensor is shaken more when the pectoral muscle on the side of the implant is active than when there is counterbalancing by symmetrical activity of the upper torso as when both arms are lifted or when lifting with the opposite arm.

The variation in the response of the two types of activity sensing pacemaker, as well as being the result of differing programming recommendations by the manufacturers, may also be the result of different processing of the sensor signal. The piezo crystal, which is bonded to the can in the Sensolog unit, and the activity sensors in both DDDR units that we studied, respond more to pressure than vibration because processing takes account of amplitude, frequency, and total variation in signal length. In the Activitrax pacemaker the sensor is also can-bonded but responds more to can vibration, with the piezo crystal acting partly as a vibration counter, and less to pressure over the pacemaker itself. ${ }^{28}$ This may make the Activitrax less specific than the Sensolog but the programming algorithms seem to allow better simulation of the natural chronotropic response to a wider variety of daily activities with the Activitrax than with the Sensolog.

The Meta unit, which senses minute ventilation, and Vitatron $\mathrm{Tx}$, which senses the stimulus-QT interval, proved slow to react to burst exercise and tended to underrespond to most protocols. Similar findings were recently reported with these pacemakers during the performance of daily activities. ${ }^{29}$ The Tx units often responded so slowly to stress-for example staircase ascent and descent and walking deceleration-that rates increased paradoxically in the recovery phases. This slowness to respond is the result of the changes in the stimulus-QT interval at the start of exercise. Baig et al showed an acute prolongation of the stimulus-QT interval at the start of treadmill exercise before the widely accepted shortening after more prolonged stress. ${ }^{30}$ Despite improvements in detection algorithms and rate response slopes this finding will make it difficult for this type of sensor to respond rapidly and accurately to everyday burst activity. Patients with Vitatron $\mathrm{Tx}$ units, however, responded best of all the VVIR systems to the mental stress protocol, closely simulating the natural chronotropic response. This confirms that functioning of this sensor was more "physiological", with the response based on a probable increase in adrenergic drive during the protocol; this ultimately shortened the stimulus-QT interval giving a good simulation of the normal chronotropic response to resting stress.

The Meta patients underresponded to mental stress and change in posture. They were slow to respond when they walked up a staircase and remained in high rate response well into the recovery period, though their response to walking down stairs was appropriate. This sensor lacks specificity because an increase in heart rate does not always correlate with an increase in either respiratory rate or minute ventilation. ${ }^{26}$ Thus though there was significantly more response to resting stress than that found with the activity sensors it was significantly less than in the controls. The persistence of high heart rates during recovery phases paralleled the increased respiratory rate which decays more slowly than heart rate in controls.

Other workers found a paradoxical change in pacing rate in patients implanted with Meta units when they performed the Valsalva manoeuvre while supine. ${ }^{31} \mathrm{An}$ increase in rate with arm swinging was also reported with VVIR units sensing minute ventilation and units sensing respiratory rate such as the Biotec Biorate $^{32}$; we did not find that this was an important effect in our studies.

The dual chamber pacemakers all tended to simulate the natural chronotopic response during standardised daily activities. In our study the Medico Phymos VDD units underresponded to mental stress. This was because in two patients heart rate increased sufficiently to activate the rate limiting function that was programmed to avoid pacemaker mediated tachycardia by inducing a 2:3 heart block at rates over 150 beats per minute. This may have biased the group result and it is therefore difficult to draw firm conclusions from these data.

Most patients implanted with DDDR pacemakers in this study had both sinus node dysfunction and high grade atrioventricular block. This system, therefore, is the only one we have assessed where a "combination" sensor is operating by tracking the sinus response and using an activity sensor to augment the rate response and thus "support" the sinus node when its response is inadequate. During mental stress when the activity sensor is not contributing to the rate adaptation, the tracking response increases heart rate although significantly less than in controls. The activity sensor, however, seems to predominate during the staircase descent protocol, causing an initial overresponse to this stress. Overall, however, these units performed better than any of the single sensor, single chamber rate adaptive pacemakers we studied.

In all but one patient with a DDD pacemaker 
in our study group, the response of the sinus node to exercise was normal. Overall simulation of the normal chronotropic response during the everyday activity protocols was best in patients with DDD pacemakers.

CONCLUSION

All the pacemakers under investigation showed appropriate heart rate response to exercise treadmill testing when compared with healthy controls. Our data show that the simulation of natural chronotropic response during daily activities is generally poor in patients implanted with VVIR pacemakers. The advantages and disadvantages of different sensors depend on their specificity and sensitivity to increased physiological demand.

A combination sensor may improve the rate adaptation of single chamber rate responsive pacemakers during everyday activity. Early rate adaptation to physical stress by sensors that rapidly respond to activity followed by later input by "physiological" sensors (that is those that respond to minute ventilation, stimulus to QT interval, $\mathrm{pH}$, temperature, or oxygen saturation) would improve pacemaker sensitivity and specificity to a wide variety of stresses and facilitate better simulation of the normal sinus node response to everyday activities and circadian variations in heart rate. ${ }^{33}$ Recent work with multiple sensors seems promising. ${ }^{34}$

At present, dual chamber pacemakers still give the best form of rate responsive pacing in terms of heart rate changes during everyday activities.

1 Alt E, Volker R, Wirtzfeld A, Ulm K. Survival and followup after pacemaker implantation: a comparison of patien with sick sinus syndrome, complete heart block and atria fibrillation. $P A C E$ 1985;8:849-55.

2 Rickards AF, Donaldson RM. Rate responsive pacing. Clin Prog Pacing Electrophysiol 1983;1:12-9.

3 Karlof I Haemodynamic effect of atrial triggered versus fixed rate pacing at rest and during exercise in complete heart block. Acta Med Scand 1975;197:195-203.

4 Rossi P, Rognoni G, Occhetta E, et al. Respiration dependent ventricular pacing compared with fixed ventricular dent ventricular pacing compared with fixed ventricular and A-V synchronous pacing: aerobic and haem

5 Beyersdorf F, Kreuzer J, Happ J, Zegelman M, Satter P. Increase in cardiac output with rate responsive pace Increase in cardiac output with rate resp

6 Humen DP, Kostuk WS, Klein GJ. Activity sensing, rate responsive pacing: improvement in myocardial performance with exercise. $P A C E$ 1985;8:52-9.

$7 \mathrm{Lau}$ CP, Camm AJ. Role of left ventricular function and Doppler derived variables in predicting haemodynamic benefits of rate responsive pacing. Am J Cardiol 1988;62:906-11.

8 Humen DP, Anderson K, Brumwell D, et al. A pacemaker which automatically increases its rate with physical activity. In: World symposium of cardiac pacing: proceedings. Dormstadt, Federal Republic of Germany: Steinkopff Verlag, 1983:259-64.

9 Rossi P, Plicchi G, Canducci GC, Rognoni G, Aina F. Respiratory rate as a determinant of optimal pacing rate. PACE 1983;6:502-10.
10 Rickards AF, Donaldson RM, Thalen HJ. The use of QT interval to determine pacing rate: early clinical experience. PACE 1983;6:346-54

11 Alt E, Hirgstetter C, Heinze M, Blomer H. Rate control of physiologic pacemakers by central venous blood temperature. Circulation 1986;73:1206-12.

12 Wirtzfeld A, Goedel-Meinen L, Bock T, Heinze R, Liss $\mathrm{HD}$, Munteanu J. Central venous oxygen saturation for the control of automatic rate responsive pacing. $P A C E$ 1982;5:829-35.

13 Cammilli L, Alcidi L, Papeschi G, et al. Preliminary experience with $\mathrm{pH}$-triggered pacemaker. $P A C E$ 1978; $1: 448-57$

14 Sutton R, Sharma A, Ingram A, Camm J, Lindemans F, Bennett $T$. First derivative of right ventricular pressure as a sensor for an implantable rate response VVI pacemaker [Abstract]. PACE 1988;11:487.

15 Callaghan F, Scamerlo J, Tarjarn P. The ventricular depolarisation gradient: exercise performance of a closed loop rate responsive pacemaker [Abstract]. $P A C E$ 1987;10:1212

16 Kenny RA, Ingram A, Mitsouka $T$, Walsh $\mathrm{K}$, Sutton $\mathrm{R}$. Comparison of sensor driven physiological pacing systems [Abstract]. PACE 1985;8:781.

17 Alt E, Volker R, Hogl B, MacCarter D. Comparison of Activitrax and Nova MR pacemakers with VVI/AAI pacing. Circulation 1988;78 (suppl III):116-24.

18 Maisch B, Langenfeld $\mathrm{H}$. Rate adaptive pacing-clinical experience with 3 different pacing systems. PACE 1986;9:997-1004.

19 Ausubel K, Furman S. The pacemaker syndrome. Ann Intern Med 1985;103:420-9.

20 Wilkoff BL, Corey J, Blackburn C. Analytic techniques in the assessment of chronotropic response to exercise [Abstract]. PACE 1988;11:530.

21 Lipkin DP, Buller N, Frenneaux M, et al. Randomised crossover trial of rate responsive Activitrax and conventional fixed rate ventricular pacing. Br Heart J 1987 58:613-6

22 Faerestrand S, Breivik K, Ohm O. Assessment of the work capacity and relationship between rate response and exercise tolerance associated with activity sensing rate responsive ventricular pacing. $P A C E$ 1987;10:1277-90.

23 Nordlander R, Pehrsson SK, Astron H, Karlsson J. Myocardial demands of atrial-triggered versus fixed rate ventricular pacing in patients with complete heart block. $P A C E$ 1987;10:1154-9.

24 Sharma A, Bennett T, Sutton R, Ericson M, Yee R, Klein G. Randomised single blind assessment of rate responsive pacing based upon maximum positive right ventricular pacing based upon maximum positive right ventricular
$\mathrm{dP} / \mathrm{dt}$ during treadmill exercise [Abstract]. $P A C E$ 1988;11:487.

25 Ryden L, Kristenssen B-E. Rate responsive pacing: effects on haemodynamics, exercise tolerance, and subjective feeling of wellbeing. In: Zipes DP, Jalife J, eds. Rate responsive pacing. New York: Grune, 1985:513-22.

26 Rossi P. Rate responsive pacing: biosensor reliability and physiological sensitivity. PACE 1987;10:454-66.

27 Lau CP, Mehta D, Toff WD, Stott RJ, Ward DE, Camm AJ. Limitations of rate response of an activity-sensing rate responsive pacemaker to different forms of activity. PACE 1988;11:141-6.

28 Gillette $\mathrm{P}$. Implantable sensor for closed-loop prosthetic systems. In: Ko WH, Mugica J, Ripart A, eds. Critical analysis of sensors for physiological rate responsive pacing. Mount Kisco, NY: Futura, 1985:251-6.

29 Lau CP, Wong CK, Leung WH, Cheng CH, Lo CW. A comparative evaluation of minute ventilation sensing and activity sensing adaptive-rate pacemakers during daily activity sensing adaptive-rate pace

30 Baig W, Sutcliffe R, Green A, Perrins CJ. Initial experience with slope adaptation in the Tx 919 QT sensing pacemaker with slope adaptation in the Tx $919 \mathrm{QT}$ sensing pacemaker [Abstract]. In: Proceedings of 4th sym

31 Alt E, Heinz M, Hirgstetter C, Emslader HP, Daum S Blomer $\mathrm{H}$. Control of pacemaker rate by impedance based respiratory minute ventilation. Chest 1987;92:247-52.

32 Occhetta E, Prando MD, Perucca A, et al. Respiratory pacemaker dysfunction: 4 years follow-up. In: Santini $M$ Pistolese M, Alliegro A, eds. Progress in clinical pacing. Rome, 1986:77.

33 Wertheimer L, Hassen AZ, Delman AJ. The 24 hour (circadian) rhythm of the cardiovascular system. Clin Res 1972;20:404-11.

34 Stangl K, Wirtzfeld A, Heinze R, et al. A new multisenso pacing system using stroke volume, respiratory rate, mixed venous oxygen saturation, and temperature, right atrial pressure, right ventricular pressure, and $\mathrm{dP} / \mathrm{dt}$. PACE 1988;11:712-24. 